\title{
Tratamientos térmicos
}

\author{
Heat treatments \\ Mariana Becerra-Rodríguez, ${ }^{a}$,Vanessa J. Aguilar-Díaz ${ }^{b}$, Jaqueline Bernardino-González $^{c}$, \\ Fernando Santana-Ramírez ${ }^{d}$
}

\begin{abstract}
:
In this work shown, concise information is presented about the concept of heat treatment and its different types. The present theoret ical base proposes the correct way to define the types of thermal treatments that exist, at the same time that it briefly explains the process in which these methods are carried out; as well as the different applications in the industrial environment, which we could notice thanks to the records about changes in the behaviour of materials when they are submitted to different temperatures, making modifications in their initial mechanical properties, adapting them to any work. Improving the life of the processed material, making it optimal for each type of work that requires it. Demonstrating that there is a relationship between the treatment of a material and how this causes improvements in the resistance, ductility, hardness and other properties of metals. With this we seek to provide this information to university students as well as professionals in different branches of knowledge, hoping that this knowledge will be useful in working life.
\end{abstract}

Keywords:

Temperature, property, modification

\section{Resumen:}

En este trabajo mostrado, se presenta información concisa acerca de él concepto de tratamiento térmico y sus diferentes tipos. La presente base teórica se propone la forma correcta para definir los tipos de tratamientos térmicos que existen, a la par de explicar brevemente el proceso en el que se llevan a cabo dichos métodos; así como las distintas aplicaciones en el medio industrial, que pudimos notar gracias a los registros acerca de cambios en el comportamiento de los materiales al ser sometidos a distintas temperaturas, haciendo modificaciones en sus propiedades mecánicas iniciales adecuándolo para cualquier labor. Mejorando la vida útil del material procesado, haciéndolo óptimo para cada tipo de trabajo que lo requiera. Demostrando que existe una relación entre el tratamiento de un material y cómo este causan mejoras en la resistencia, ductilidad, dureza y otras propiedades de los metales. Con esto buscamos facilitar esta información a los estudiantes universitarios, así como a profesionales en distintas ramas del saber, esperando que este conocimiento les sea de provecho en la vida laboral.

Palabras Clave:

Temperatura, propiedad, modificación

\section{Introducción}

\subsection{Los tratamientos térmicos}

Se definen como el modo de mejorar la resistencia, ductilidad, dureza y algunas otras propiedades de los metales a aquellos procesos que involucran temperatura, es decir, es la operación de calentamiento o enfriamiento de un metal en su estado sólido a temperaturas y condiciones determinadas para mejorar sus propiedades mecánicas. Producir estos cambios se debe gracias a la generación de ciertas condiciones en el calentamiento,

\footnotetext{
autor de correspondencia, Universidad Autónoma del Estado de Hidalgo, https://orcid.org/0000-0002-0879-1877, Email: be353623@uaeh.edu.mx

b Universidad Autónoma del Estado de Hidalgo,https://orcid.org/0000-0002-6001-1014, Email: ag352506@uaeh.edu.mx c Universidad Autónoma del Estado de Hidalgo, https://orcid.org/0000-0001-7182-9973,Email: be420962@uaeh.edu.mx 
permanencia o enfriamiento de los materiales tratados. El objetivo de todos los tratamientos térmicos es lograr que los cambios estructurales se produzcan en las condiciones deseadas.

Para realizar un tratamiento térmico se deben conocer las propiedades del metal en sus diversos estados estructurales. [1]

\subsection{Tipos de tratamientos}

\section{Templado}

Este procedimiento se hace a temperaturas elevadas de $300^{\circ} \mathrm{C}$ a $500^{\circ} \mathrm{C}$, esto para endurecer el acero lo cual también es conocido como temple o templado. En este procedimiento es de mucha importancia el tiempo de su calentamiento y enfriamiento para poder llegar a la dureza que se requiere. [2]

La temperatura fluye del exterior del acero al interior para hacerlo a una determinada velocidad, porque también depende del tamaño del acero. El tiempo de calentamiento es mayor que el tratamien to conocido como normalizado para permitir un endurecimiento homogéneo. Los aceros se someten a carbonización y algunas aleaciones con $0.35 \%$ de contenido de carbono, hace que el material pueda llegar a soportar cargas dinámicas sin quebrarse. [2]

Para llevar a cabo el enfriamiento en algunos aceros de bajo y medio contenido de carbono el procedimiento se hace con agua y cuando son con alto contenido de carbono generalmente de hace con aceite.

\section{Revenido}

Los aceros posteriores al temple suelen quedar generalmente demasiado duros y frágiles para los diferentes usos a los que van a ser destinados. Estos inconvenientes se corrigen por medio del tratamiento térmico del revenido. [3]

El término revenido hace referencia a aquel proceso industrial en el que se emplean bajas temperaturas como se menciona más adelante, y posteriormente deján dolo enfriar. Dependiendo del material en el que se apliquen estos procedimientos se puede variar la temperatura del revenido entre 160 y $500{ }^{\circ} \mathrm{C}$ o superior. Se realiza normalmente después de un proceso de temple, con el objetivo de alcanzar la proporción de du reza y resistencia deseada. [3]

Normalmente este tratamiento se lleva a cabo en homos de revenido, que se pueden equipar con gas protector opcional. El gas impedirá que la superficie del material se oxide durante el proceso. Para algunos tipos de acero, el tiempo de mantenimiento a la temperatura de revenido es de gran importancia, dependiendo del grado de acero y a determinados intervalos de temperatura puede producirse un fenómeno conocido como fragilización por revenido, el cual es irreversible, ocurre en un rango aproximado entre $250-400^{\circ} \mathrm{C}$ y se denomina a menudo como "fragilidad azul". [3]

Dentro de las principales ventajas de éste tratamiento se encuentra que la dureza del material se reduce y a cambio aumenta la solidez, lo permite adaptar dichas propiedades (relación dureza/resistencia) para una aplicación específica.

\section{Normalizado}

Este tipo de procedimiento es similar al tratamiento de templado, pero en este caso se ocupan temperaturas mayores a $37.77^{\circ} \mathrm{C}$ al momento del revenido final, es de los procesos más sencillos en cuanto a realización. [3]

Como su nombre lo dice, es el tratamiento que se le da a los aceros para darle las características necesarias para considerarlos "normales". Es utilizado para piezas fundidas, forjadas o mecanizadas y sirve para afinar su estructura, mejorar la maquinabilidad y eliminar tensiones que se forman al momento de solidificación.

Un tipo de tratamiento como este se lleva acabo a una temperatura alta fantes mencionada), seguida de un enfriamiento con aire quieto hasta llegar a una temperatura ambiente. El objetivo de este tipo de tratamiento es aumentar la dureza del acero con el que se esté trabajando.

\section{Recocido}

Es un procedimiento en el que se busca que los materiales tratados pierdan dureza y ganen flexibilidad, eliminando las tensiones creadas durante el temple. El proceso de recocido se lo puede utilizar para eliminar totalmente el endurecimiento que se causa por deformación que se consigue durante el proceso de trabajo en frío; este componente final será blando y dúctil. [4]

El proceso consiste en calentar el material a temperaturas elevadas hasta el punto de austenización $\left(800^{\circ} \mathrm{C}-950^{\circ} \mathrm{C}\right.$ dependiendo del material) seguido de un enfriamiento más lento. El trabajo se realiza en vacío o en una atmósfera reductora si se quiere un acabado brillante, al aire para elementos que requieran una mecanización posterior o en una atmósfera neutra donde no habrá descarburación, aunque el acabado no será brillante. [4] Esta transformación facilita el mecanizado, da estabilidad dimensional y se produce una mejora en las propiedades mecánicas y eléctricas del material.

\subsection{Aplicaciones de los tipos de tratamientos}

Templado:

Al aluminio se le puede aplicar este tratamiento térmico ya que este de manera pura es blando y tiene poca 
resistencia con este tratamiento el aluminio puede tener una serie de propiedades más resistentes para el uso comercial e industria. [5]

Revenido:

Este tratamiento se utiliza en aceros para herramientas de trabajo frio y calientes dado a que estas herramientas necesitan alrededor de las 60 unidades Rockwell (HRC) hablando de herramientas frías y de las 300 unidades Brinell (HB) y 65 HRC para las herramientas d trabajo caliente, otra aplicación que tiene este tratamiento es el acero para muelles ya que estos requieren una dureza de los 45 HRC. [6]

Normalizado:

Se lleva a cabo en aceros al carbón y acercaros de baja aleación, la laminación en caliente o la fundición, esto ya que este tratamiento térmico aumenta la dureza. La dureza depende del "análisis dimensional del acero y la velocidad de enfriamiento". [7]

\section{Recocido:}

Principalmente a los productos que por lo general se le aplican este tratamiento térmico son semiacabados como piezas de forja, piezas de fundición o laminas al igual que otras ejecuciones en frio, al igual el acabado queda brillante en una atmosfera reductora y en una atmosfera neutral no será brillante. [7] 


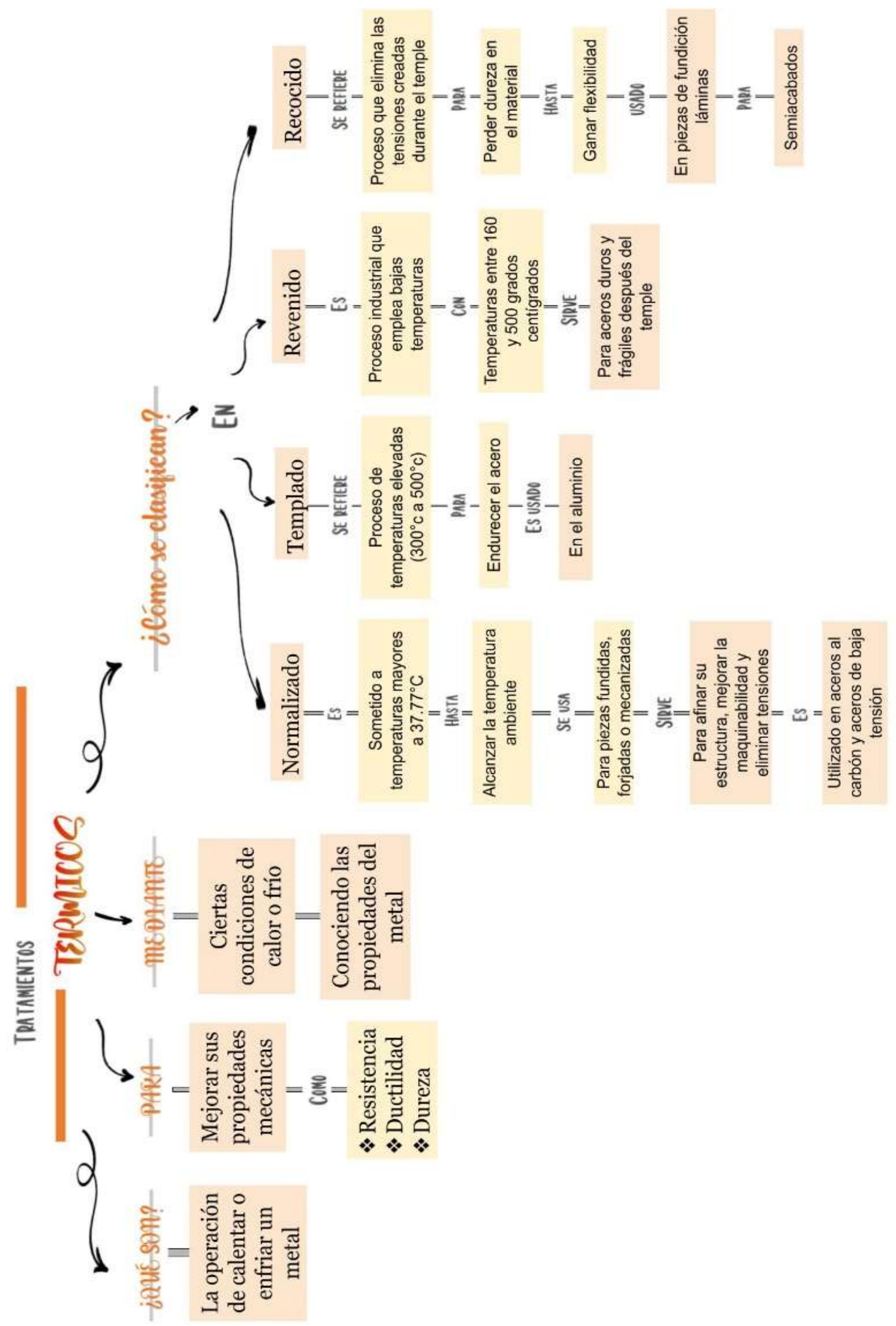




\section{Agradecimientos}

Le agradecemos a nuestro catedrático, la Dra. Lizeth Martínez Ayala, adscrita a la Escuela superior Tepeji de la Universidad Autónoma del estado de Hidalgo, por guiarnos y asesorarnos en todo el proceso que conllevó este proyecto, sin lo anterior esto no hubiera sido posible.

\section{Referencias}

[1] Introducción a los tratamientos térmicos - Metalmecánica. (n.d.). Retrieved August 25, 2020, from https://www.interempresas.net/MetalMecanica/Articulos/2506Introduccion-a-los-tratamientos-termicos.html

[2] Juan, I., \& Pérez, A. (2017). Universidad Autónoma de Nuevo León Facultad de Ingeniería Mecánica y Eléctrica que presenta el M M.

[3] Restrieved August 25, (2020) Evaluación de la dureza, Tenacidad y Microestructura en un acero Sae 1045 Templado En Soluciones De Salmuera Con Posterior Revenido. From Http://Bibliotecas.Unsa.Edu.Pe/Bitstream/Handle/UNSA/10523/Imchu rlf.Pdf?Sequence=1\&Isallowed $=Y$

[4] López, A. (2013) "Recocido materialen la creación de herramientas de prueba" (tesis de obtención de título) Universidad Politécnica Salesiana Sede Quito Carrera: Ingeniería Mecánica

[5] Benjamin García, A de J (2010) Temple industriales Alcala from: https://www.templesindustrialesalcala.es/tratamientos-termicos/

[6] Juaez, A. (2020) Revenido - Temple y revenido - Tratamiento térmico Bodycote Plc. (n.d.), from https://www.bodycote.com/es/servicios/tratamiento-termico/temple-yrevenido/revenido/

[7] August G. (2020) Tratamiento térmico Recocido - Temples industriales Alcalá. (n.d.). Retrieved, from https://www.templesindustrialesalcala.es/recocido/\#: :text=Los productos a los que, clasificarse según la temperatura aplicada. 